\title{
An Improved Backstepping-Based Controller for Three-Dimensional Trajectory Tracking of a Midwater Trawl System
}

\author{
Zhao Yan, ${ }^{1}$ Yun sheng Mao, ${ }^{2}$ and Cheng gang $\mathrm{Liu}^{3}$ \\ ${ }^{1}$ Key Laboratory of High Performance Ship Technology (Wuhan University of Technology), Ministry of Education, Wuhan, China \\ ${ }^{2}$ Wuhan University of Technology, Wuhan, China \\ ${ }^{3}$ China Ship Development and Design Center, Wuhan, China \\ Correspondence should be addressed to Zhao Yan; yanzhao19890907@163.com
}

Received 9 April 2016; Accepted 11 August 2016

Academic Editor: William MacKunis

Copyright (c) 2016 Zhao Yan et al. This is an open access article distributed under the Creative Commons Attribution License, which permits unrestricted use, distribution, and reproduction in any medium, provided the original work is properly cited.

\begin{abstract}
An improved backstepping control method for three-dimensional trajectory tracking of a midwater trawl system is investigated. A new mathematical model of the trawl system while considering the horizontal expansion effect of two otter boards is presented based on the Newton Euler method. Subsequently, an active path tracking strategy of the trawl system based on the backstepping method is proposed. The nonstrict feedback characteristic of the proposed model employs a control allocation method and several parallel nonlinear PID (Proportion Integration Differentiation) controllers to eliminate the high-order state variables. Then, the stability analysis by the Lyapunov Stability Theory shows that the proposed controller can maintain the stability of the trawl system even with the presence of external disturbances. To validate the proposed controller, a simulation comparison with a linear PID controller was conducted. The simulation results illustrate that the improved backstepping controller is effective for three-dimensional trajectory tracking of the midwater trawl system.
\end{abstract}

\section{Introduction}

Single-boat midwater trawl systems have occupied an important position in pelagic fisheries. These systems consist of a fishing vessel, two sets of warp and otter boards, a towing winch system, and a trawl net. The entire system is difficult to control because the length of the warp connecting the fishing vessel and the trawl net could be thousands of meters, and the warps and trawl net are flexible. Generally during fishing operations, the adjustment of the trawl system motion state primarily depends on the experience of the sailors. To improve the capture efficiency, the concept of automatic fishing is proposed, which utilizes a controller to track the trajectory of the trawl net based on the motion model of the entire system.

As a complicated underwater towing system, the dominant feature of a trawl system is the flexibility of the trawl net and warps. Park [1] assumed the trawl net shape from the wing end to the bag net to be an elliptic cone. The dynamics of the warps can be described by various mathematical models [2-4]. When the motion models of these subsystems are integrated as a whole, the system theoretically has infinite order, which means the trajectory tracking control is difficult to achieve. Hence, the motion mathematical model of the trawl system should be greatly simplified.

First, Umeda presented a first-order model and proposed an optimal method to realize the depth regulation of the trawl net [5]. Hu et al. proposed the control input for a depth controller by analyzing the motion of a midwater trawl system [6]. Then, the PID controller was developed. Johansen et al. considered the horizontal movement of the trawl system [7]. Reite and Sorensen installed and experimented with an actuator on the otter board to control the trawl depth [8]. The research employed a multiple PID controller to regulate the movement of the trawl net via the otter board [9]. Afterwards, several nonlinear models of the trawl system were proposed 
to improve the depth control accuracy. Lee et al. presented a fuzzy controller and provided experiment results [10, 11]. Zhou et al. proposed a robust optimal output tracking control method based on a T-S fuzzy nonlinear model that makes the trawl net rapidly follow the desired trajectory under model uncertainties and external disturbances $[12,13]$. Furthermore, a great deal of research has been devoted to the trajectory tracking of underwater towed systems [14-17], which are similar to the depth control of trawl systems.

The trajectory tracking control of a trawl system is in three-dimensional space; therefore, in addition to the depth adjustment, the horizontal-position control of the trawl net is needed. Coordinated work between the tow winch and the ship motion control is required. The state space model of a trawl system could be high-order nonlinear. In this case, backstepping control is a common recursive design method in nonlinear control theory. This method can avoid the error caused by the linearization of a nonlinear system. This method has been widely used in the trajectory tracking control of complex underactuated systems. For ship tracking control, $\mathrm{Fu}$ et al. considered the trajectory tracking of a vessel with only the measurable position and heading angle, and the author designed a nonlinear backstepping observer controller [18]. For an unmanned underwater vehicle (UUV), $\mathrm{Xu}$ et al. proposed a trajectory tracking controller based on a backstepping method and proposed a virtual velocity error dynamic method instead of an attitude error dynamic method to avoid the singularity problem [19]. Wang et al. designed a path-following controller for UUV in threedimensional space through backstepping method and eliminated part of the nonlinear coupled terms for the simplification of the virtual control [20]. Zhu et al. combined a traditional backstepping model with a bioinspired neurodynamics model and developed a three-dimensional UUV-trajectory controller [21, 22]. For the Unmanned Aerial Vehicle (UAV), Khebbache proposed a control algorithm based on robust integral backstepping considering the actuator faults [23]. Fang et al. presented a double-loop control scheme and designed an adaptive backstepping controller for small-scale unmanned helicopters [24].

In this paper, the trajectory tracking problem of a midwater trawl system in three-dimensional space is considered. Compared to the standard simplified model used in $[12,13]$, a new simplified model to express the dynamics of the trawl system while considering the horizontal motion of two otter boards is presented. This new model can fully embody the horizontal expansion of the trawl net provided by the two otter boards. Subsequently, a three-dimensional trajectory tracking controller of the trawl system is proposed based on the backstepping control approach. Compared to the previous backstepping method, a compound nonlinear controller is adopted to solve the problem that decoupling is difficult for multiple high-order variables in the nonlinear trawl system. Based on the Lyapunov Stability Theory, the proposed control approach is proved with good robustness and stability under external disturbance. Finally, the effectiveness and superiority of the proposed control approach are illustrated by numerical simulation.

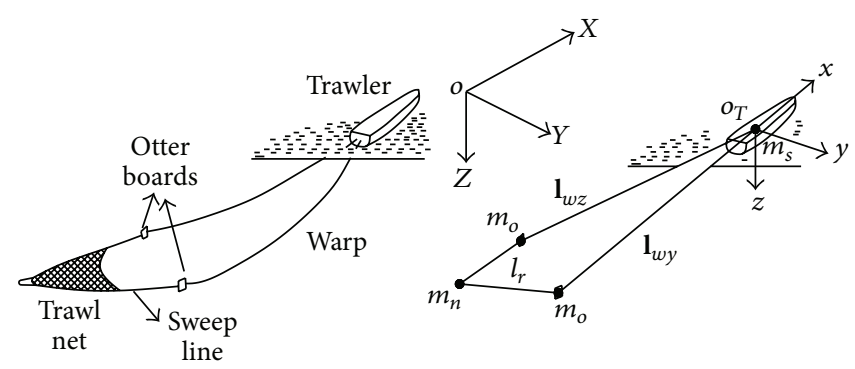

FIGURE 1: Simplified trawl system.

\section{Three-Dimensional Kinematic Model of the Trawl System}

A trawl system is a combination of a rigid body and a flexible body. Generally, the motion of the trawl system in three-dimensional space should have 6 degrees of freedom. However, based on existing control theory, this trajectory tracking problem can hardly be solved. In addition, the position vector of the trawl net is the most important information in fishing operations. Hence, this work only considers the parallel motion in 3 degrees of freedom.

Previous researchers have proposed a simplified model to exploit the tracking problems of a trawl system within the vertical plane $[12,13]$. In this work, the model has been improved for application to the space tracking problem. As shown in Figure 1, the two otter boards, the vessel, and the trawl net are simplified into lumped mass points. These four mass points are connected through two warps and two sweeps.

For the purpose of reducing the amount of calculations, the simplified model is developed based on the Newton Euler method. The location of the model can be described by two coordinate systems, which are the earth coordinates $(X, Y, Z)$ and the ship fixed coordinates $(x, y, z)$. The warps and sweeps are considered to be elastic links. The tensions of these ropes can be calculated by Hooke's law.

The trawler movement can be described as $X$-direction movement $u, Y$-direction movement $v$, and yawing $P$ in the earth coordinates. The dynamic equations of marine vessels [25] are referenced, and the 3 DOF kinetic equations of the trawler can be written as

$$
\begin{aligned}
M_{s} \ddot{q}_{s}+B_{s} \dot{q}_{s}+C_{s} \dot{q}_{s}= & k_{2}^{s z}\left(q_{s}-q_{o z}-\mathbf{1}_{w z}\right) \\
& +k_{2}^{s y}\left(q_{s}-q_{o y}-\mathbf{1}_{w y}\right)+T_{s},
\end{aligned}
$$

where $q_{s}=\left[X_{s}, Y_{s}, \varphi\right]^{T}$ is the position vector of the trawler; $q_{o z}$ and $q_{o y}$ are the position vectors of the two otter boards; $\mathbf{l}_{w z}$ and $\mathbf{l}_{w y}$ are the lengths of the two warps, and they are also vectors. $T_{s}$ is the thrust of the vessel produced by the propeller and rudder, which is the control vector. $k_{2}^{s z}=E_{w} A_{w} / \mathbf{l}_{w z}$ is the elasticity coefficient of the port warp, and $k_{2}^{s y}$ is for the starboard warp. When deploying the warp, the warp material velocity and the boundary node velocity on the winch are inconsistent; this velocity contrast can generate a thrust force [16]. In this work, the warp is considered to be an elastic link; this thrust force can hardly be calculated. Hence we assume 
that the payout speed of the winch is slow enough so that the velocity contrast can be very low. Comparing with the large physical sizes of the whole system and a long simulation time (thousands of seconds), the thrust force caused by the velocity contrast of the warp and the winch can be ignored.

For the parameter matrixes, they can be written as

$$
\begin{aligned}
M_{s} & =M R^{T}(\varphi), \\
C_{s} & =\left[C(v)-M R^{T}(\varphi) \dot{R}(\varphi)\right] R^{T}(\varphi), \\
B_{s} & =B R^{T}(\varphi),
\end{aligned}
$$

where $M, C$, and $B$ are the inertial matrix, the Coriolis force matrix, and the damping force matrix of the trawler, respectively. And they are all defined in the ship fixed coordinates $(x, y, z)$. The expressions of $M, C$, and $B$ can be found in [26]. $R(\varphi)$ is the coordinate transform matrix. It can be expressed as

$$
R(\varphi)=\left[\begin{array}{ccc}
\cos \varphi & -\sin \varphi & 0 \\
\sin \varphi & \cos \varphi & 0 \\
0 & 0 & 0
\end{array}\right]
$$

The kinetic equations of the trawl winch on the port side can be expressed as

$$
J_{w} \ddot{\mathbf{i}}_{w z}=W_{d}-B_{w} \dot{\mathbf{l}}_{w z}-T_{w} \cdot r_{w}
$$

where $q_{o z}=\left[X_{q z}, Y_{q z}, Z_{q z}\right]^{T}$ is the position vector of the port otter board. $J_{w}$ is the rotational inertia of the winch, it can be written as $J_{w}=J_{w o}+\mu L r_{w o}^{2}+\mu d\left(L^{2}-l^{2}\right) / 2 \pi n$, and the meanings of these symbols can be found in Table 2. $W_{d}=p_{L} D_{w}$ is the output torque of the winch motor. $p_{L}$ and $D_{w}$ are the load pressure and the motor displacement, respectively. $B_{w}$ is the damping coefficient of the winch, and $T_{w}$ is the tension of the warp. Since the warp is considered to be elastic link (refer to (1)), $T_{w}$ can be expressed as $T_{w}=$ $k_{2}^{s z}\left(q_{s}-q_{o z}-\mathbf{1}_{w z}\right) \cdot r_{w}$ is the radius of the roller.

The otter board provides the expansionary forces to hold the horizontal width of the trawl net. Figure 2 is the schematic diagram of the otter board. Based on D'Alembert's principle, the kinetic equations of the otter board in the earth coordinates can be expressed as

$$
\begin{aligned}
& m_{o} \ddot{q}_{o z}+B_{o}\left|\dot{q}_{o z}\right| \dot{q}_{o z}+W_{o} \\
& \quad=k_{2}^{s z}\left(q_{s}-q_{o z}-\mathbf{1}_{w z}\right)-k_{1}^{s}\left(q_{o z}-q_{n}-\mathbf{1}_{r z}\right),
\end{aligned}
$$

where $q_{n}=\left[\begin{array}{lll}X_{q n} & Y_{q n} & Z_{q n}\end{array}\right]^{T}$ is the position vector of the trawl net. $k_{1}^{s}=E_{r} A_{r} / l_{r}$ and $\mathbf{l}_{r z}$ are the elasticity coefficient and the length vector of the sweep, respectively. $m_{o}$ and $W_{o}$ are the equivalent mass and the gravity of the otter board, respectively. $B_{o}=1 / 2 \rho C_{d o} S_{o}$ is the resistance coefficient of the otter board. $\rho$ is the fluid density, and $C_{d o}$ and $S_{o}$ are the hydrodynamic coefficient and the area of the otter board, respectively. Obviously, the hydrodynamic performance of the otter board is decided by its attitude (attack and slip angles). Hence $C_{d o}$ is varying with the movement of the otter board, but in order to simplify the calculation, a constant coefficient $C_{o}$ is used to substitute $C_{d o}$. And the added mass of the otter board is ignored since its physical dimensions are much less than the trawler. The equations of the two otter boards are basically the same.

Similar to the otter board, the kinetic equations of the trawl net can be expressed as

$$
\begin{aligned}
& m_{n} \ddot{q}_{n}+B_{n}\left|\dot{q}_{n}\right| \dot{q}_{n}+W_{n} \\
& \quad=k_{1}^{s}\left(q_{o z}-q_{n}-\mathbf{1}_{r z}\right)+k_{1}^{s}\left(q_{o y}-q_{n}-\mathbf{1}_{r y}\right),
\end{aligned}
$$

where $m_{n}$ and $W_{n}$ are the equivalent mass and the gravity of the trawl net, respectively. $B_{n}=1 / 2 \rho C_{d n} S_{n}$ is the damping coefficient of the trawl net. Similar to $C_{d o}$, a constant coefficient $C_{n}$ is used to substitute $C_{d n}$.

Thus, the three-dimensional kinematic model of the trawl system can be described by (1), (4), (5), and (6). The state vector of this model can be defined as $x=\left[\begin{array}{llllllllllll}q_{n} & \dot{q}_{n} & q_{o z} & \dot{q}_{o z} & q_{o y} & \dot{q}_{o y} & q_{s} & \dot{q}_{s} & \mathbf{l}_{w z} & \dot{\mathbf{l}}_{w z} & \mathbf{l}_{w y} & \mathbf{i}_{w y}\end{array}\right]$. The control inputs of this model contain the ship propulsion system and two trawl winches, which can be defined as $u=\left[\begin{array}{lll}u_{s} & u_{w z} & u_{w y}\end{array}\right]$. Then, the state space equations of the trawl system can be expressed as

$$
\begin{aligned}
& \dot{x}_{1}=x_{2}, \\
& \dot{x}_{2}=\frac{\left[-\widehat{B}_{n} \cdot x_{2}^{2}-W_{n}+k_{1}^{s}\left(x_{3}-x_{1}-\mathbf{1}_{r z}\right)+k_{1}^{s}\left(x_{5}-x_{1}-\mathbf{1}_{r y}\right)+w_{n}\right]}{m_{n}}, \\
& \dot{x}_{3}=x_{4}, \\
& \dot{x}_{4}=\frac{\left[-\widehat{B}_{o} \cdot x_{4}^{2}-W_{o}-k_{1}^{s}\left(x_{3}-x_{1}-\mathbf{1}_{r z}\right)+k_{2}^{s z}\left(x_{7}-x_{3}-x_{9}\right)+w_{o z}\right]}{m_{o}}, \\
& \dot{x}_{5}=x_{6}, \\
& \dot{x}_{6}=\frac{\left[-\widehat{B}_{o} \cdot x_{6}^{2}-W_{o}-k_{1}^{s}\left(x_{5}-x_{1}-1_{r y}\right)+k_{2}^{s y}\left(x_{7}-x_{5}-x_{11}\right)+w_{o y}\right]}{m_{o}},
\end{aligned}
$$




$$
\begin{aligned}
& \dot{x}_{7}=x_{8}, \\
& \dot{x}_{8}=\frac{\left[u_{s}-B_{s} \cdot x_{8}-C_{s} \cdot x_{8}+k_{2}^{s z}\left(x_{7}-x_{3}-x_{9}\right)+k_{2}^{s y}\left(x_{7}-x_{5}-x_{11}\right)\right]}{M_{s}}, \\
& \dot{x}_{9}=x_{10}, \\
& \dot{x}_{10}=\frac{\left[u_{w z}-B_{w} \cdot x_{10}-k_{2}^{s z}\left(\left|x_{7}-x_{3}\right|-x_{9}\right) \cdot r_{w}\right]}{J_{w}}, \\
& \dot{x}_{11}=x_{12}, \\
& \dot{x}_{12}=\frac{\left[u_{w y}-B_{w} \cdot x_{12}-k_{2}^{s y}\left(\left|x_{7}-x_{5}\right|-x_{11}\right) \cdot r_{w}\right]}{J_{w}},
\end{aligned}
$$

where $w_{o}$ and $w_{n}$ are the interference forces on the otter boards and the trawl net.

\section{Three-Dimensional Trajectory Tracking Control Approach}

The control objective is to make the trawl net move along the target trajectory, which is defined as $x_{1 d}$. There are 12 components in state vector $x$; thus, the virtual control inputs of all of the state variables can be defined as $x_{2 d} \sim x_{12 d}$. Then, the tracking error of the trawl system can be written as

$$
\begin{gathered}
z_{1}=x_{1}-x_{1 d} \\
\vdots \\
z_{12}=x_{12}-x_{12 d} .
\end{gathered}
$$

3.1. Controller Design. The midwater trawl system is a highorder nonlinear system. To obtain the control instruction $u$ of the whole system, the state space model is divided into six subsystems. The principle of system division is that the lower-order subsystem stabilization relies on the next higherorder subsystem. From the target trajectory $x_{1 d}$ to the control instruction $u$, the six subsystems are based on the dynamic equations of the trawl net, the two otter boards, the trawler, and the two trawl winches, respectively. Based on the physical relations of the six subsystems, Figure 3 shows the demand relationships of the key virtual control inputs. The Lyapunov functions, which are related to the control objectives, can then be built with the following steps.

Step 1. For the first subsystem, the Lyapunov function is given by

$$
V_{1}=\frac{1}{2} z_{1}^{T} z_{1}
$$

The time derivative of $V_{1}$ is

$$
\dot{V}_{1}=z_{1}^{T} \dot{z}_{1}=z_{1}^{T}\left(x_{2 d}+z_{2}-\dot{x}_{1 d}\right) .
$$

The virtual control inputs $x_{2 d}$ can be defined as

$$
x_{2 d}=-k_{1} z_{1}+\dot{x}_{1 d},
$$

where the control parameter $k_{1} \geq 0$. Equation (11) is plugged into (10), and the derivative of $V_{1}$ is

$$
\dot{V}_{1}=-k_{1} z_{1}^{T} z_{1}+z_{1}^{T} z_{2}
$$

Step 2. For the second subsystem, the Lyapunov function is given by

$$
V_{2}=V_{1}+\frac{1}{2} z_{2}^{T} z_{2}
$$

The time derivative of $V_{2}$ is

$$
\dot{V}_{2}=\dot{V}_{1}+z_{2}^{T} \dot{z}_{2}=\dot{V}_{1}+z_{2}^{T}\left[\frac{\left(-\widehat{B}_{o} x_{2}^{2}-W_{n}+k_{1}^{s}\left(x_{3}-x_{1}-\mathbf{1}_{r z}\right)+k_{1}^{s}\left(x_{5}-x_{1}-\mathbf{1}_{r y}\right)+w_{n}\right)}{m_{o}}-\dot{x}_{2 d}\right] .
$$

The traditional backstepping method can only be applied to the strict feedback form of nonlinear systems. However, in (14), the high-order state variable $x_{5}$ appears. Thus a new variable is introduced, which is defined as $x_{s o}=x_{3}+x_{5}$. The new virtual control inputs $x_{\text {sod }}$ can be embodied as

$$
x_{\text {sod }}=\frac{\left(m_{n} \dot{x}_{2 d}+\widehat{B}_{n} x_{2}^{2}+W_{n}+2 k_{1}^{s} x_{1}-m_{n} z_{1}+k_{1}^{s}\left(\mathbf{1}_{r z}+\mathbf{l}_{r y}\right)-m_{n} k_{2} z_{2}\right)}{k_{1}^{s}}
$$




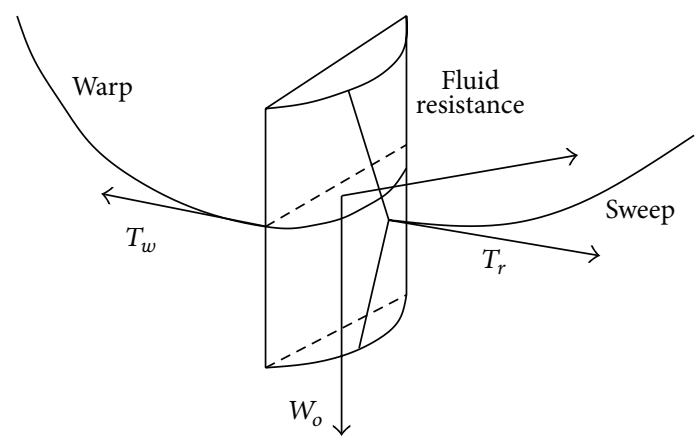

FIGURE 2: Diagrammatic sketch of otter board.

where the control parameter $k_{2} \geq 0$. Equation (15) is plugged into (14), and the derivative of $V_{2}$ is

$$
\dot{V}_{2}=-k_{1} z_{1}^{T} z_{1}-k_{2} z_{2}^{T} z_{2}+\frac{k_{1}^{s}}{m_{n}} z_{2}^{T} z_{s o}+z_{2}^{T} \frac{w_{n}}{m_{n}},
$$

where $z_{s o}=z_{3}+z_{5}$.

The variables $x_{3 d}$ and $x_{5 d}$ are the ideal position vectors of the two otter boards. To deduce their expressions from $x_{\text {sod }}$, these two otter boards are assumed to move at the same depth underwater such that $x_{3 d}=0.5 x_{\text {sod }}$ and $x_{5 d}=0.5 x_{\text {sod }}$ in the $z$-direction. In addition, assuming that the extension ratios of the ropes are consistent in three directions, the variables $x_{3 d}$ and $x_{5 d}$ in the $x$-and $y$-directions can be expressed as

$$
\begin{aligned}
x_{3 d}(y)= & \frac{x_{3 d}(z)-x_{1 d}(z)-\mathbf{1}_{r z}(z)}{\mathbf{1}_{r z}(z)} \mathbf{1}_{r z}(y)+x_{1 d}(y) \\
& +\mathbf{l}_{r z}(y) .
\end{aligned}
$$

Step 3. For the third subsystem, the Lyapunov function is given by

$$
V_{3}=V_{2}+\frac{1}{2} z_{3}^{T} z_{3}+\frac{1}{2} z_{5}^{T} z_{5}
$$

The time derivative of $V_{3}$ is

$$
\dot{V}_{3}=\dot{V}_{2}+z_{3}^{T}\left(z_{4}+x_{4 d}-\dot{x}_{3 d}\right)+z_{5}^{T}\left(x_{6 d}+z_{6}-\dot{x}_{5 d}\right) \text {. }
$$

The two virtual variables can be defined as

$$
\begin{aligned}
& x_{4 d}=\dot{x}_{3 d}-k_{3} z_{3}-\frac{k_{1}^{s}}{m_{n}} z_{2}, \\
& x_{6 d}=\dot{x}_{5 d}-k_{5} z_{5}-\frac{k_{1}^{s}}{m_{n}} z_{2},
\end{aligned}
$$

where the control parameters $k_{3} \geq 0, k_{5} \geq 0$. Equation (20) is plugged into (19), and the derivative of $V_{3}$ is

$$
\begin{aligned}
\dot{V}_{3}= & -k_{1} z_{1}^{T} z_{1}-k_{2} z_{2}^{T} z_{2}-k_{3} z_{3}^{T} z_{3}-k_{5} z_{5}^{T} z_{5}+z_{2}^{T} \frac{w_{n}}{m_{n}} \\
& +z_{3}^{T} z_{4}+z_{5}^{T} z_{6} .
\end{aligned}
$$

Step 4. For the fourth subsystem, the Lyapunov function can be defined as

$$
V_{4}=V_{3}+\frac{1}{2} z_{4}^{T} z_{4}+\frac{1}{2} z_{6}^{T} z_{6}
$$

The time derivative of $V_{4}$ is

$$
\begin{aligned}
\dot{V}_{4}= & \dot{V}_{3}+z_{4}^{T}\left[\frac{\left(-\widehat{B}_{o} x_{4}^{2}-W_{o}-k_{1}^{s}\left(x_{3}-x_{1}-\mathbf{1}_{r z}\right)+k_{2}^{s z}\left(x_{7}-x_{3}-x_{9}\right)+w_{o z}\right)}{m_{o}}\right] \\
& +z_{6}^{T}\left[\frac{\left(-\widehat{B}_{o} x_{6}^{2}-W_{o}-k_{1}^{s}\left(x_{5}-x_{1}-\mathbf{1}_{r y}\right)+k_{2}^{s y}\left(x_{7}-x_{5}-x_{11}\right)+w_{o y}\right)}{m_{o}}\right] .
\end{aligned}
$$

In (23), the high-order state variables $x_{9}$ and $x_{11}$ appeared. Similar to Step 2, new variables can be introduced as

$$
\begin{aligned}
& x_{s z}=x_{7}-x_{9}, \\
& x_{s y}=x_{7}-x_{11} .
\end{aligned}
$$

The corresponding virtual control inputs can be defined as

$$
\begin{aligned}
& x_{s z d}=x_{7 d}-x_{9 d}, \\
& x_{s y d}=x_{7 d}-x_{11 d} .
\end{aligned}
$$

For one vector $x_{7 d}$, two expressions can be derived from (25). Unfortunately, the tensions of the two warps are not equal when the vessel is turning, which leads to a conflict in solving $x_{7 d}$. Moreover, (25) is based on the physical interpretation of force and displacement; however, the physical interpretation of $x_{7 d}$ is the $x$-coordinate, the $y$ coordinate, and the yawing angle of the vessel on the target trajectory. Therefore, the physical meaning of (25) in the $z$ direction is not correct.

To obtain the high-order control outputs $x_{7 d}, x_{9 d}$, and $x_{11 d}$, a multiple nonlinear PID approach is adopted. Take $x_{7 d}$, for example; the trajectory tracking error of the trawl net can be used as control inputs. Then, the output of controller for $x_{7 d}$ can be expressed as

$$
\begin{aligned}
x_{7 d}(t)= & k_{p}\left(z_{1}(t)\right) z_{1}(t)+k_{i}\left(z_{1}(t)\right) \int_{0}^{t} z_{1}(t) d t \\
& +k_{d}\left(z_{1}(t)\right) \frac{d z_{1}(t)}{d t}
\end{aligned}
$$




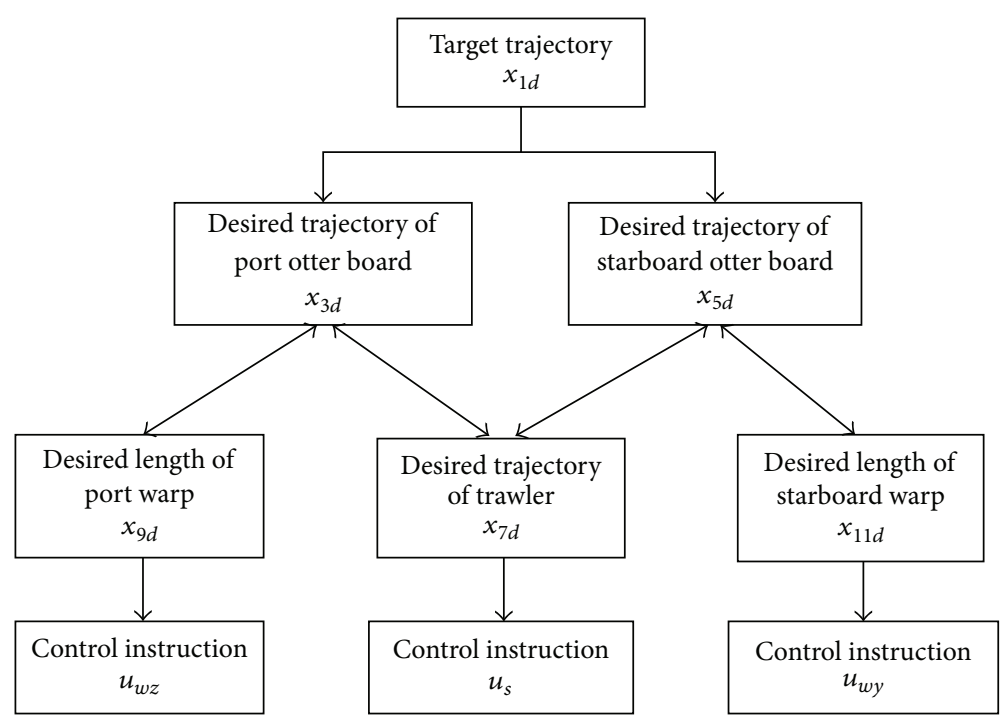

Figure 3: Physical connection of key state variables.

where the control parameters are the nonlinear functions of $z_{1}(t)$. These time-varying parameters can be written as

$$
\begin{aligned}
& k_{p}\left(z_{1}(t)\right)=a_{p}+b_{p}\left(1-\operatorname{sech}\left(c_{p} z_{1}(t)\right)\right), \\
& k_{d}\left(z_{1}(t)\right)=a_{d}+\frac{b_{d}}{\left(1+c_{d} \exp \left(d_{d} z_{1}(t)\right)\right)}, \\
& k_{i}\left(z_{1}(t)\right)=a_{i} \operatorname{sech}\left(c_{i} z_{1}(t)\right),
\end{aligned}
$$

where $a_{p}, b_{p}, c_{p}, a_{d}, b_{d}, c_{d}, d_{d}, a_{i}$, and $c_{i}$ are all positive constants.

Considering the physical significance of $x_{9 d}$ and $x_{11 d}$, the deviation between the actual tension and the ideal tension of the warps can be used as control inputs. The controller outputs for $x_{9 d}$ and $x_{11 d}$ are similar to (27).

Plugging the expressions of these variables into (23), the derivative of $V_{4}$ can be found by

$$
\begin{aligned}
\dot{V}_{4}= & -k_{1} z_{1}^{T} z_{1}-k_{2} z_{2}^{T} z_{2}-k_{3} z_{3}^{T} z_{3}-k_{4} z_{4}^{T} z_{4}-k_{5} z_{5}^{T} z_{5} \\
& -k_{6} z_{6}^{T} z_{6}+\frac{k_{2}^{s z}}{m_{o}} z_{4}^{T} z_{s z}+\frac{k_{2}^{s y}}{m_{o}} z_{6}^{T} z_{s y} .
\end{aligned}
$$

Step 5. For the fifth subsystem, the Lyapunov function is given by

$$
V_{5}=V_{4}+\frac{1}{2} z_{7}^{T} z_{7}+\frac{1}{2} z_{9}^{T} z_{9}+\frac{1}{2} z_{11}^{T} z_{11}
$$

The time derivative of $V_{5}$ is

$$
\begin{aligned}
\dot{V}_{5}= & \dot{V}_{4}+z_{7}^{T}\left(x_{8 d}+z_{8}-\dot{x}_{7 d}\right) \\
& +z_{9}^{T}\left(x_{10 d}+z_{10}-\dot{x}_{9 d}\right) \\
& +z_{11}^{T}\left(x_{12 d}+z_{12}-\dot{x}_{11 d}\right) .
\end{aligned}
$$

Three virtual variables can be defined as

$$
\begin{aligned}
& x_{8 d}=\dot{x}_{7 d}-k_{7} z_{7}-\frac{k_{2}^{s z}}{m_{o}} z_{4}-\frac{k_{2}^{s y}}{m_{o}} z_{6}, \\
& x_{10 d}=\dot{x}_{9 d}-k_{9} z_{9}+\frac{k_{2}^{s z}}{m_{o}} z_{4}, \\
& x_{12 d}=\dot{x}_{11 d}-k_{11} z_{11}+\frac{k_{2}^{s y}}{m_{o}} z_{6},
\end{aligned}
$$

where the control parameters $k_{7} \geq 0$, and $k_{9} \geq 0, k_{11} \geq 0$. Plugging (31) into (30), the derivative of $V_{5}$ is found as

$$
\begin{aligned}
\dot{V}_{5}= & -k_{1} z_{1}^{T} z_{1}-k_{2} z_{2}^{T} z_{2}-k_{3} z_{3}^{T} z_{3}-k_{4} z_{4}^{T} z_{4}-k_{5} z_{5}^{T} z_{5} \\
& -k_{6} z_{6}^{T} z_{6}-k_{7} z_{7}^{T} z_{7}-k_{9} z_{9}^{T} z_{9}-k_{11} z_{11}^{T} z_{11} \\
& +z_{2}^{T} \frac{w_{n}}{m_{n}}+z_{4}^{T} \frac{w_{o}}{m_{o}}+z_{6}^{T} \frac{w_{o}}{m_{o}}+z_{7}^{T} z_{8}+z_{9}^{T} z_{10} \\
& +z_{11}^{T} z_{12} .
\end{aligned}
$$

Step 6. For the last subsystem, the Lyapunov function is written as

$$
V_{6}=V_{5}+\frac{1}{2} z_{8}^{T} z_{8}+\frac{1}{2} z_{10}^{T} z_{10}+\frac{1}{2} z_{12}^{T} z_{12}
$$

The time derivative of $V_{6}$ is 


$$
\begin{aligned}
\dot{V}_{6}= & \dot{V}_{5}+z_{8}^{T}\left[\frac{\left(u_{s}-B_{s} x_{8}-C_{s} x_{8}+k_{2}^{s z}\left(x_{7}-x_{3}-x_{9}\right)+k_{2}^{s y}\left(x_{7}-x_{5}-x_{11}\right)\right)}{M_{s}}-\dot{x}_{8 d}\right] \\
& +z_{10}^{T}\left[\frac{\left(u_{w z}-B_{w} x_{10}-k_{2}^{s z}\left(\left|x_{7}-x_{3}\right|-x_{9}\right) r_{w}\right)}{J_{w}}-\dot{x}_{10 d}\right] \\
& +z_{12}^{T}\left[\frac{\left(u_{w y}-B_{w} x_{12}-k_{2}^{s y}\left(\left|x_{7}-x_{5}\right|-x_{11}\right) r_{w}\right)}{J_{w}}-\dot{x}_{12 d}\right] .
\end{aligned}
$$

The controlled quantities of the trawler and two winches can be defined as

$$
\begin{aligned}
u_{s}= & M_{s} \dot{x}_{8 d}+B_{s} x_{8}+C_{s} x_{8}-k_{2}^{s z}\left(x_{7}-x_{3}-x_{9}\right) \\
& -k_{2}^{s y}\left(x_{7}-x_{5}-x_{11}\right)-k_{8} z_{8}-M_{s} z_{7}, \\
u_{w z}= & J_{w} \dot{x}_{10 d}+B_{w} x_{10}+k_{2}^{s z}\left(\left|x_{7}-x_{3}\right|-x_{9}\right) r_{w} \\
& -k_{10} z_{10}-J_{w} z_{9}, \\
u_{w y}= & J_{w} \dot{x}_{12 d}+B_{w} x_{12}+k_{2}^{s y}\left(\left|x_{7}-x_{5}\right|-x_{11}\right) r_{w} \\
& -k_{12} z_{12}-J_{w} z_{11},
\end{aligned}
$$

where the control parameters $k_{8} \geq 0$, and $k_{10} \geq 0, k_{12} \geq 0$. Thus, the three-dimensional trajectory tracking controller of trawl system has been acquired.

3.2. Stability Analysis. To indicate the trawl system stability under the bounded input signal and external environment disturbance, three disturbance terms are added to the state model. Then, the expressions of $\dot{x}_{2}, \dot{x}_{4}$, and $\dot{x}_{6}$ in (7) can be rewritten as

$$
\begin{aligned}
& \dot{x}_{2}=\frac{\left[-\widehat{B}_{n} \cdot x_{2}^{2}-W_{n}+k_{1}^{s}\left(x_{3}-x_{1}-\mathbf{1}_{r z}\right)+k_{1}^{s}\left(x_{5}-x_{1}-\mathbf{1}_{r y}\right)+w_{n}\right]}{m_{n}}, \\
& \dot{x}_{4}=\frac{\left[-\widehat{B}_{o} \cdot x_{4}^{2}-W_{o}-k_{1}^{s}\left(x_{3}-x_{1}-\mathbf{1}_{r z}\right)+k_{2}^{s z}\left(x_{7}-x_{3}-x_{9}\right)+w_{o z}\right]}{m_{o}}, \\
& \dot{x}_{6}=\frac{\left[-\widehat{B}_{o} \cdot x_{6}^{2}-W_{o}-k_{1}^{s}\left(x_{5}-x_{1}-\mathbf{1}_{r y}\right)+k_{2}^{s y}\left(x_{7}-x_{5}-x_{11}\right)+w_{o y}\right]}{m_{o}},
\end{aligned}
$$

where $w_{n}, w_{o z}$, and $w_{o y}$ are the disturbing forces on the trawl net and two otter boards.

For the condition that nonzero bounded disturbances often exist in trawl system, the Lyapunov function can be defined as

$$
V_{7}=V_{6}+z_{2}^{T} \frac{w_{n}}{m_{n}}+z_{4}^{T} \frac{w_{o z}}{m_{o}}+z_{6}^{T} \frac{w_{o y}}{m_{o}} .
$$

According to the inequality $z_{2}^{T} w_{n} / m_{n} \leq\left(a z_{2}^{2}+\right.$ $\left.\left\|w_{n}\right\|^{2} /(4 a)\right) / m_{n}$, the time derivative of $V_{7}$ satisfies the following inequality:

$$
\begin{aligned}
\dot{V}_{6} \leq & -k_{1} z_{1}^{T} z_{1}-\left(k_{2}-\frac{a}{m_{n}}\right) z_{2}^{T} z_{2}-k_{3} z_{3}^{T} z_{3} \\
& -\left(k_{4}-\frac{b}{m_{n}}\right) z_{4}^{T} z_{4}-k_{5} z_{5}^{T} z_{5} \\
& -\left(k_{6}-\frac{c}{m_{n}}\right) z_{6}^{T} z_{6}-k_{7} z_{7}^{T} z_{7}-k_{8} z_{8}^{T} z_{8} \\
& -k_{9} z_{9}^{T} z_{9}-k_{10} z_{10}^{T} z_{10}-k_{11} z_{11}^{T} z_{11}-k_{12} z_{12}^{T} z_{12} \\
& +\frac{\left\|w_{n}\right\|^{2}}{4 a m_{n}}+\frac{\left\|w_{o}\right\|^{2}}{4 b m_{o}}+\frac{\left\|w_{o}\right\|^{2}}{c b m_{o}} .
\end{aligned}
$$

The definite integrals of (36) can be expressed as

$$
\begin{aligned}
& V\left(x\left(t_{f}\right)\right)-V(x(0)) \\
& \quad \leq \rho^{2} \int_{0}^{t_{f}} w^{T}(t) w(t) d t-\int_{0}^{t_{f}} z^{T}(t) z(t) d t
\end{aligned}
$$

where $\rho^{2}=\operatorname{diag}\left(1 / 4 a m_{n} 1 / 4 b m_{o} 1 / 4 c m_{o}\right), w=$ $\left[\begin{array}{lll}w_{n} & w_{o z} & w_{o y}\end{array}\right]$, and $z=\operatorname{diag}\left(k_{1}, k_{2}-a / m_{n}, k_{3}, k_{4}-b / m_{o}, k_{5}\right.$, $\left.k_{6}-c / m_{o}, k_{7}, \ldots, k_{12}\right)\left[z_{1}, z_{2}, \ldots, z_{12}\right]$.

Through (39) it can be seen that the tracking error is global uniform ultimate boundedness, and the state of the system converges to the origin of a small domain in the stable area in the presence of interference with an unknown upper limit.

\section{Simulation Experiment}

The simulation experiment was conducted based on the MATLAB/Simulink platform. The trawl system is assumed to move in the uniform flow. The parameters of the trawl system are listed in Tables 1 and 2 . 
TABLE 1: Trawler, otter board, and trawl net parameters.

\begin{tabular}{lcc}
\hline Quantity & Symbol & Value \\
\hline Mass of trawler & $m_{s}($ ton $)$ & 5718 \\
Mass of otter board & $m_{o}($ ton $)$ & 5.2 \\
Mass of trawl net & $m_{o}($ ton $)$ & 47.916 \\
Area of otter board & $S_{o}\left(\mathrm{~m}^{2}\right)$ & 12 \\
Area of trawl net & $S_{n}\left(\mathrm{~m}^{2}\right)$ & 2107 \\
$\begin{array}{l}\text { Resistance coefficient of trawl net } \\
\text { Resistance coefficient of otter }\end{array}$ & $C_{n}$ & 1.5 \\
board & $C_{o}$ & 0.5 \\
$\begin{array}{l}\text { Otter board weight in water } \\
\text { (Z-direction) }\end{array}$ & $W_{o}(\mathrm{~N})$ & 45520 \\
$\begin{array}{l}\text { Trawl net weight in water } \\
\text { (Z-direction) }\end{array}$ & $W_{n}(\mathrm{~N})$ & 15826 \\
$\begin{array}{l}\text { Area of rudder } \\
\text { Resistance deduction fraction of } \\
\text { rudder }\end{array}$ & $S_{r}\left(\mathrm{~m}^{2}\right)$ & 12 \\
$\begin{array}{l}\text { Thrust deduction fraction of } \\
\text { trawler }\end{array}$ & $t_{r}$ & 0.236 \\
$\begin{array}{l}\text { Diameter of propeller } \\
\text { Propeller revolution rate } \\
\text { Thrust coefficient under standard } \\
\text { pitch ratio }\end{array}$ & $t_{p}$ & \\
Coefficient of pitch ratio & $D_{p}(\mathrm{~m})$ & 0.143 \\
\hline & $n_{p}(\mathrm{r} / \mathrm{min})$ & 155 \\
$K_{\mathrm{TA}}$ & 0.446 \\
$C_{p}$ & 0.568 \\
\hline
\end{tabular}

TABLE 2: Warp, sweep line, and trawl winch model parameters.

\begin{tabular}{lcc}
\hline Quantity & Symbol & Value \\
\hline Tether length of warp & $L_{\max }(\mathrm{m})$ & 3000 \\
Length of sweep line & $l_{r}(\mathrm{~m})$ & 206 \\
Diameter of warp & $d(\mathrm{~m})$ & 0.032 \\
Unit mass of warp & $\mu(\mathrm{kg} / \mathrm{m})$ & 6.3 \\
Unit length weight of warp in & $p_{w}(\mathrm{~N} / \mathrm{m})$ & 55 \\
water & $E_{w}(\mathrm{pa})$ & $10 e 9$ \\
Elasticity modulus of warp & $A_{w}\left(\mathrm{~m}^{2}\right)$ & 0.0008 \\
Cross section area of warp & $E_{r}(\mathrm{pa})$ & $10 e 9$ \\
Elasticity modulus of sweep line & $A_{r}\left(\mathrm{~m}^{2}\right)$ & 0.001 \\
Cross section area of sweep line & $r_{0}(\mathrm{~m})$ & 0.3825 \\
Initial radius of winch & $n$ & 50 \\
Turns of each layer for trawl & $n$ & 0.109 \\
winch & $D_{w}\left(\mathrm{~m}^{3}\right)$ & 150 \\
Motor displacement & $J_{w o}\left(\mathrm{~N} \cdot \mathrm{s}^{2} / \mathrm{m}\right)$ & 22 \\
Rotary inertia of winch without & $B_{w}(\mathrm{~N} \cdot \mathrm{s} / \mathrm{m})$ & 60 \\
warp & $f(\mathrm{~N})$ & \\
Damping coefficient of winch & & \\
Friction coefficient of winch &
\end{tabular}

Considering that the trawler is turning to catch a fish school, a target trajectory of the trawl net is defined as

$$
x_{1 d}=\left[\begin{array}{c}
x_{1 d}(x) \\
x_{1 d}(y) \\
x_{1 d}(z)
\end{array}\right]=\left[\begin{array}{c}
2000 \cos \left(w_{1} t-\frac{\pi}{2}\right) \\
2000 \sin \left(w_{1} t-\frac{\pi}{2}\right)+2000 \\
40 \sin \left(w_{2} t\right)-180
\end{array}\right]
$$
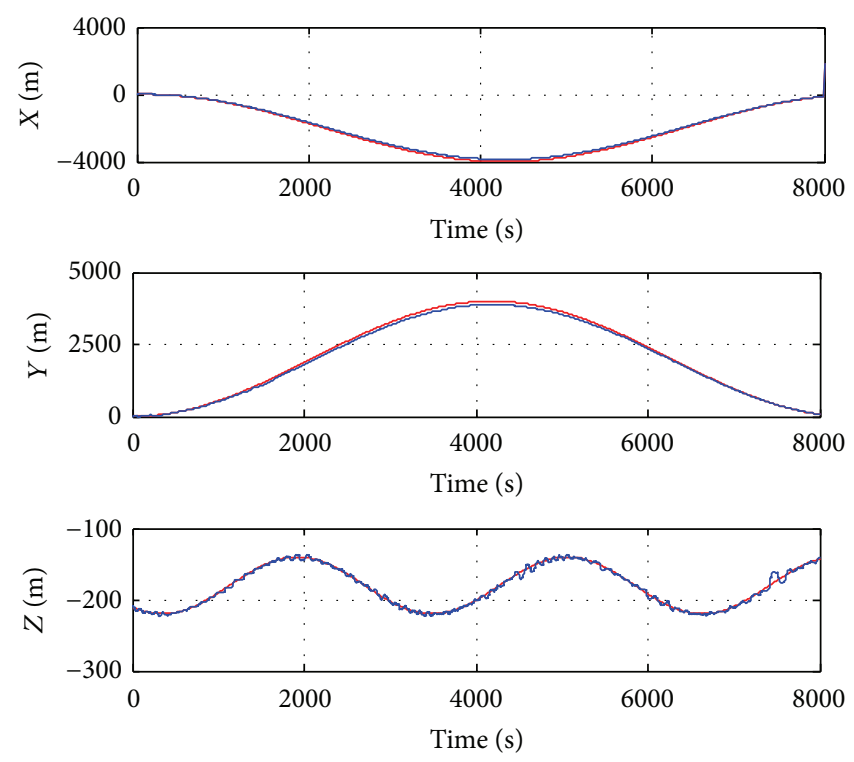

- Nominal trajectory

_ Trajectory of trawl net

FIgURE 4: Trawl system tracking trajectories.
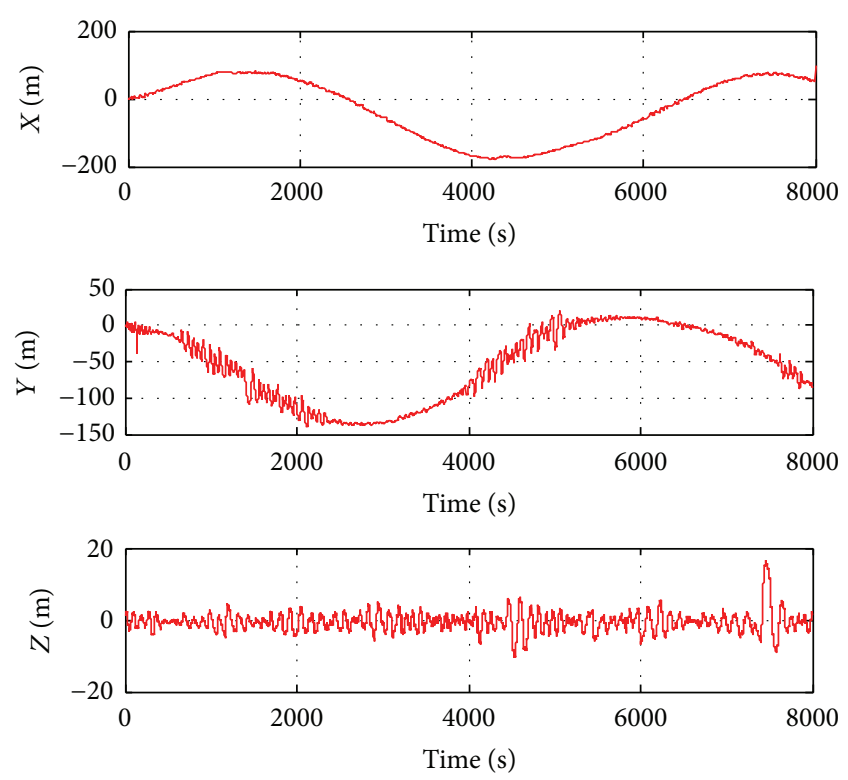

FIGURE 5: Tracking error.

where $w_{1}=0.00075 \mathrm{rad} / \mathrm{s}$ and $w_{2}=0.002 \mathrm{rad} / \mathrm{s}$. The simulation time is set as $t \in\left[\begin{array}{ll}0 & 8000\end{array}\right]$ (s).

Figure 4 shows the trajectory tracking of the trawl net when the approached control algorithm is adopted. It can be seen that the trawl net tracks the target trajectory of (40) well. Because the coordinate values of the trajectories are much larger than the tracking error, the tracking errors in three directions are individually shown in Figure 5. The mean absolute errors of tracking error in the $X$-direction, $Y$ direction, and $Z$-direction are $42.24 \mathrm{~m}, 51.36 \mathrm{~m}$, and $1.96 \mathrm{~m}$, respectively. Obviously, the tracking errors of the horizontal 

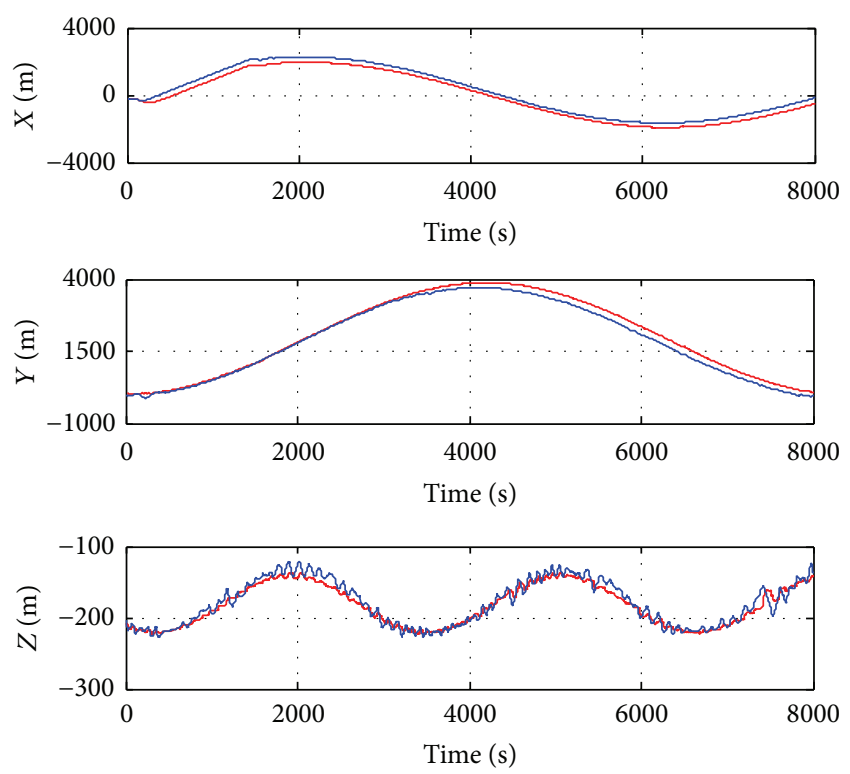

_ Trajectory of port otter board

_ Trajectory of starboard otter board

FIGURE 6: Otter board trajectories.

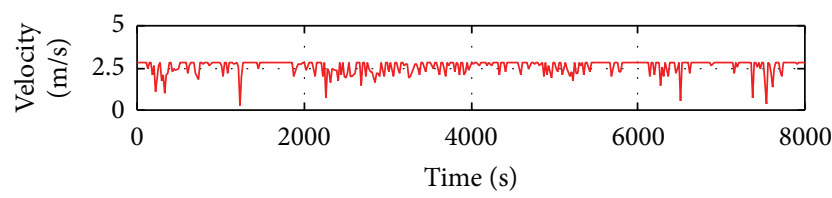

Figure 7: Trawler speed.

displacements are much larger than those of the vertical displacement, mainly because the horizontal positions of the two otter boards have no direct relation with the trawl net, and the control inputs of the otter boards in the $X$ - and $Y$ directions are derived from assumption in (17). Moreover, Figure 5 shows that the track error is cyclical; in a real-world situation, the large size of the trawl net can compensate for the tracking error in the horizontal directions.

Figure 6 shows the trajectories of the two otter boards. It can be seen that the curved shapes are similar to the trajectory of the trawl net. This movement coordination between the otter boards and the trawl net is beneficial for system stability. Figure 7 illustrates the velocity curve of the trawler. Although the trawler has frequent speed changes, the velocity is in a reasonable range. The average speed is $2.6 \mathrm{~m} / \mathrm{s}$. Figure 8 presents the dynamic changes of the lengths of the two warps. The maximum and the minimum lengths of the two warps are reasonable and bounded. Figures 9 and 10 are the thrust change curve of the trawler and the torque curves of the winches, respectively. It can be seen that these control signals have varying degrees of trembles. Compared with the response times of the ship propeller $(0.2 \mathrm{~s} \sim 1 \mathrm{~s})$ and the winch $(0.5 \mathrm{~s} \sim 2 \mathrm{~s})$, these control signals are achievable.

To further illustrate the performance of the improved backstepping method and test the robustness and adaptive

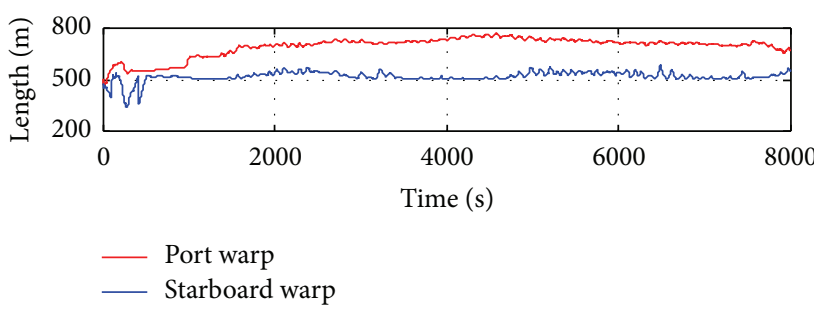

FIGURE 8: Warp lengths.

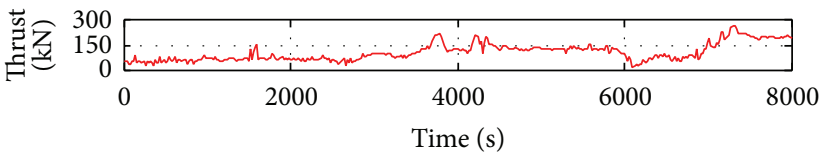

Figure 9: Thrust of the trawler.

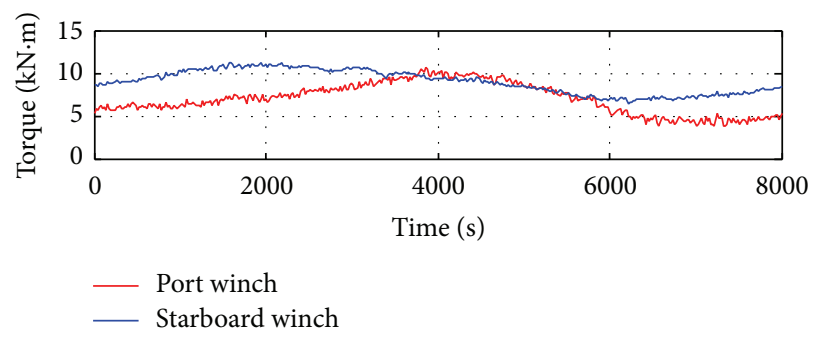

FIgURE 10: Torques of the winches.

ability of the proposed controller, a traditional linear PID controller based on the proposed simplified model is considered. A simulation contrast verification is presented while considering the interference of the external environment.

The target trajectory for the comparison simulation uses (40) with a shorter simulation time $t \in\left[\begin{array}{ll}0 & 5000\end{array}\right]$ (s). The disturbing forces $w_{o z}$ and $w_{n}$ are considered as the same constant value, which is $2.5 \times 10^{5}(\mathrm{~N})$ in the $y$-direction and $z$-direction. Respectively, $w_{o z}$ acts on the left otter board over the period of $t \in\left[\begin{array}{ll}1000 & 2000\end{array}\right](\mathrm{s})$, and $w_{n}$ acts on the trawl net over the period of $t \in\left[\begin{array}{ll}1500 & 2500\end{array}\right]$ (s). Consider that the resistance coefficients of the otter board and the trawl net are time varying, and $C_{d o}$ and $C_{d n}$ are redefined as

$$
\begin{aligned}
& C_{d o}=C_{o}\left(1+a \sin \left(w_{3} t\right)\right), \\
& C_{d n}=C_{n}\left(1+b \sin \left(w_{4} t\right)\right),
\end{aligned}
$$

where $a=0.3, b=0.35$, and $w_{3}=0.1$.

When disturbing forces are exerted on the trawl system, the trajectories of the trawl net are produced by these two controllers, as shown in Figure 11. Figure 11(a) is the simulation result of the linear PID controller, and Figure 11(b) is the simulation result of the improved backstepping controller. It can be seen that when the disturbing forces exist, the path deviation in Figure 11(a) is significantly less than the deviations in Figure 11(b), and the path deviation in the $Z$-direction is larger than the deviations in the $X$ and $Y$-directions. After the disturbing forces disappear, the path deviation is quickly eliminated under the action of 


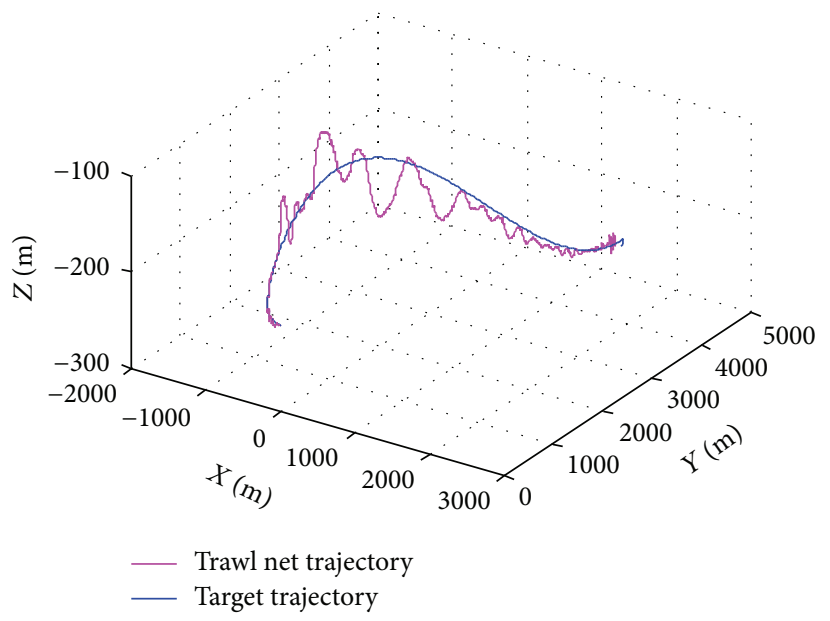

(a)

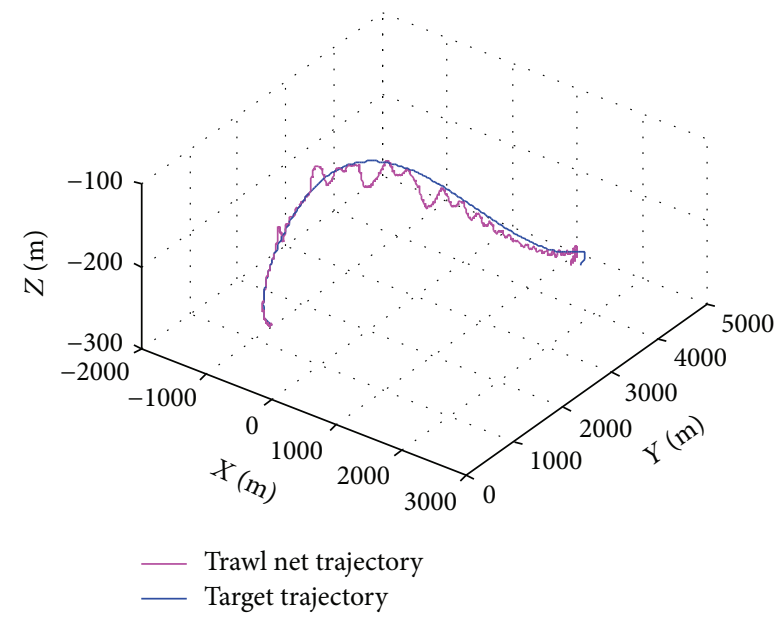

(b)

FIGURE 11: Trawl system tracking trajectories with disturbing force.

the improved backstepping controller, whereas the control process of linear PID controller is very slow. By adjusting the PID parameters, the oscillating amplitude of the path curve cannot be eliminated effectively.

\section{Conclusions}

In this paper, a three-dimensional trajectory tracking control approach of a midwater trawl system is proposed. First, according to the actual working status of a single-boat midwater trawl system, a four-material-point simplified model is presented. Second, a compound nonlinear controller is designed, based on the backstepping method. In the process of recursion, the high-order state variables are gradually eliminated. The theoretical identification and simulation illustrate the effectiveness and convergence of the proposed control method. The movement consistency of the trawl net and otter boards is displayed. Under the action of an external disturbance, the depth displacement of trawl net is larger than the horizontal displacements. Compared with the traditional linear PID control method, the improved backstepping control strategy has higher control precision and stronger robustness. In the future, an experimental demonstration will be presented.

\section{Competing Interests}

The authors declare that they have no competing interests.

\section{References}

[1] H.-H. Park, "A method for estimating the gear shape of a midwater trawl," Ocean Engineering, vol. 34, no. 3-4, pp. 470-478, 2007.

[2] C. M. Ablow and S. Schechter, "Numerical simulation of undersea cable dynamics," Ocean Engineering, vol. 10, no. 6, pp. 443-457, 1983.

[3] J. I. Gobat, M. A. Grosenbaugh, and M. S. Triantafyllou, "Generalized- $\alpha$ time integration solutions for hanging chain dynamics," Journal of Engineering Mechanics, vol. 128, no. 6, pp. 677-687, 2002.

[4] J. I. Gobat and M. A. Grosenbaugh, "Time-domain numerical simulation of ocean cable structures," Ocean Engineering, vol. 33, no. 10, pp. 1373-1400, 2006.

[5] N. Umeda, "Optimal control of a midwater trawl system," in Proceedings of the 4th Pacific Congress on Marine Science and Technology, pp. 507-514, Tokyo, Japan, July 1990.

[6] F. X. Hu, T. Tokai, and K. Matuda, "A computer simulation for the net position control of midwater trawl system," Nippon Suisan Gakkaishi, vol. 67, no. 2, pp. 226-230, 2001.

[7] V. Johansen, O. Egeland, and A. J. Sorensen, "Modelling and control of a trawl system in the transversal direction," in Control Applications in Marine Systems 2001, pp. 243-248, 2002.

[8] K. J. Reite and A. J. Sorensen, "Hydrodynamic properties important for control of trawl doors," in Proceedings of the IFAC Conference on Control Applications in Marine System (CAMS '04), pp. 143-148, Ancona, Italy, 2004.

[9] K. J. Reite, Modeling and control of trawl systems [Ph.D. thesis], Norwegian University of Science and Technology, Trondheim, Norway, 2006.

[10] L. Chun-Woo, "Depth control of a midwater trawl gear using fuzzy logic," Fisheries Research, vol. 24, no. 4, pp. 311-320, 1995.

[11] C.-W. Lee, J.-H. Lee, and I.-J. Kim, "Application of a fuzzy controller to depth control of a midwater trawl net," Fisheries Science, vol. 66, no. 5, pp. 858-862, 2000.

[12] H. Zhou, Y.-L. Chen, and H.-Y. Yang, "Robust optimal output tracking control of a midwater trawl system based on T-S fuzzy nonlinear model," China Ocean Engineering, vol. 27, no. 1, pp. $1-16,2013$.

[13] Y.-L. Chen, H. Zhou, Y.-G. Zhao, and J.-Y. Hou, "Fuzzy robust path tracking strategy of an active pelagic trawl system with coordinated ship and winch regulation," Journal of Central South University, vol. 21, no. 1, pp. 167-179, 2014.

[14] L. Chauvier, G. Damy, J. C. Gilbert, and N. Pichon, "Optimal control of a deep-towed vehicle by optimization techniques," in Proceedings of the OCEANS '98 Conference, pp. 1634-1639, IEEE, Nice, France, 1998. 
[15] P. Williams, "Towing and winch control strategy for underwater vehicles in sheared currents," International Journal of Offshore and Polar Engineering, vol. 16, no. 3, pp. 218-227, 2006.

[16] D. M. Zand, B. J. Buckham, and D. Constantinescu, "Ship and winch regulation for remotely operated vehicle's waypoint navigation," International Journal of Offshore and Polar Engineering, vol. 19, no. 3, pp. 214-223, 2009.

[17] F. C. Teixeira, A. P. Aguiar, and A. Pascoal, "Nonlinear adaptive depth tracking and attitude control of an underwater towed vehicle," in Proceedings of the 8th IFAC Conference on Manoeuvring and Control of Marine Craft, pp. 211-216, Guarujá, Brazil, September 2009.

[18] M.-Y. Fu, A.-H. Zhang, and J.-L. Xu, "Semi-global uniform exponential stable observer-controller for trajectory tracking of ships," Control and Decision, vol. 28, no. 6, pp. 920-924, 2013.

[19] J. Xu, M. Wang, and L. Qiao, "Backstepping-based controller for three-dimensional trajectory tracking of underactuated unmanned underwater vehicles," Control Theory \& Applications, vol. 31, no. 11, pp. 1589-1596, 2014.

[20] H.-J. Wang, Z.-Y. Chen, H.-M. Jia, and J. Li, “Three-dimensional path-following control of underactuated unmanned underwater vehicle using feedback gain backstepping," Control Theory \& Applications, vol. 31, no. 1, pp. 66-77, 2014.

[21] D.-Q. Zhu and R.-R. Yang, "Backstepping tracking control of autonomous underwater vehicles with bio-inspired neurdynamics model," Control Theory \& Applications, vol. 29, no. 10, pp. 1309-1316, 2012.

[22] B. Sun, D.-Q. Zhu, and Z.-G. Deng, "Bio-inspired discrete trajectory-tracking control for open-frame underwater vehicles," Control Theory \& Applications, vol. 30, no. 4, pp. 454-462, 2013.

[23] H. Khebbache, "Robust control algorithm considering the actuator faults for attitude tracking of an UAV quadrotor aircraft," International Journal of Control and Automation, vol. 5, no. 4, pp. 55-66, 2012.

[24] X. Fang, A.-G. Wu, and N. Dong, "Adaptive backstepping-based trajectory control of an unmanned helicopter in presence of wind gusts," Journal of Chinese Inertial Technology, vol. 23, no. 1, pp. 59-65, 2015.

[25] T. Fossen, Marine Control Systems: Guidance, Navigation and Control of Ships, Rigs and Underwater Vehicles, Marine Cybernetics, 2002.

[26] Y. L. Chen, Research on Modeling and Control Strategies for the Trawling System, Zhejiang University, Hangzhou, China, 2013. 


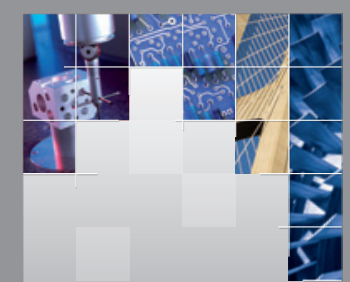

\section{Enfincering}
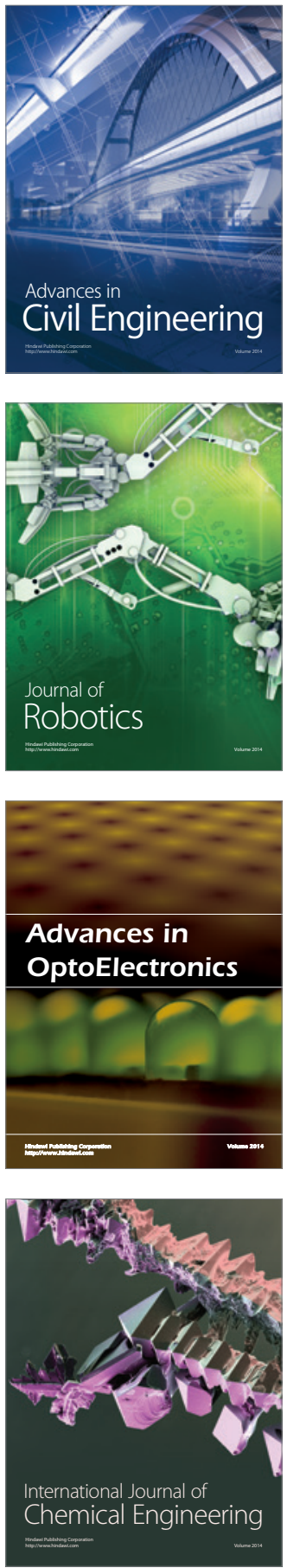

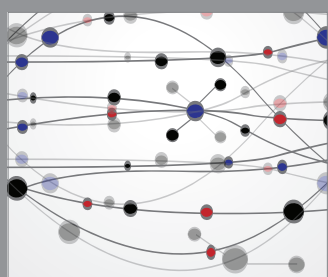

The Scientific World Journal

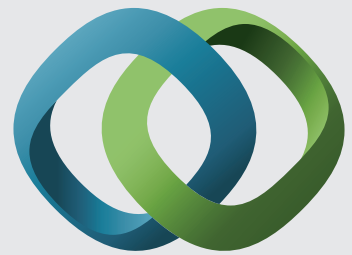

\section{Hindawi}

Submit your manuscripts at

http://www.hindawi.com
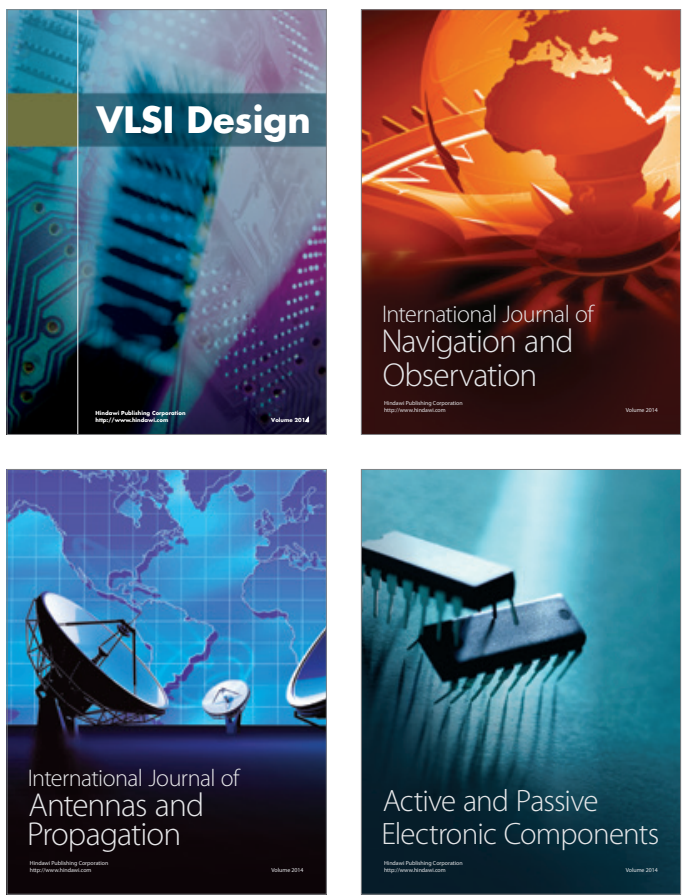
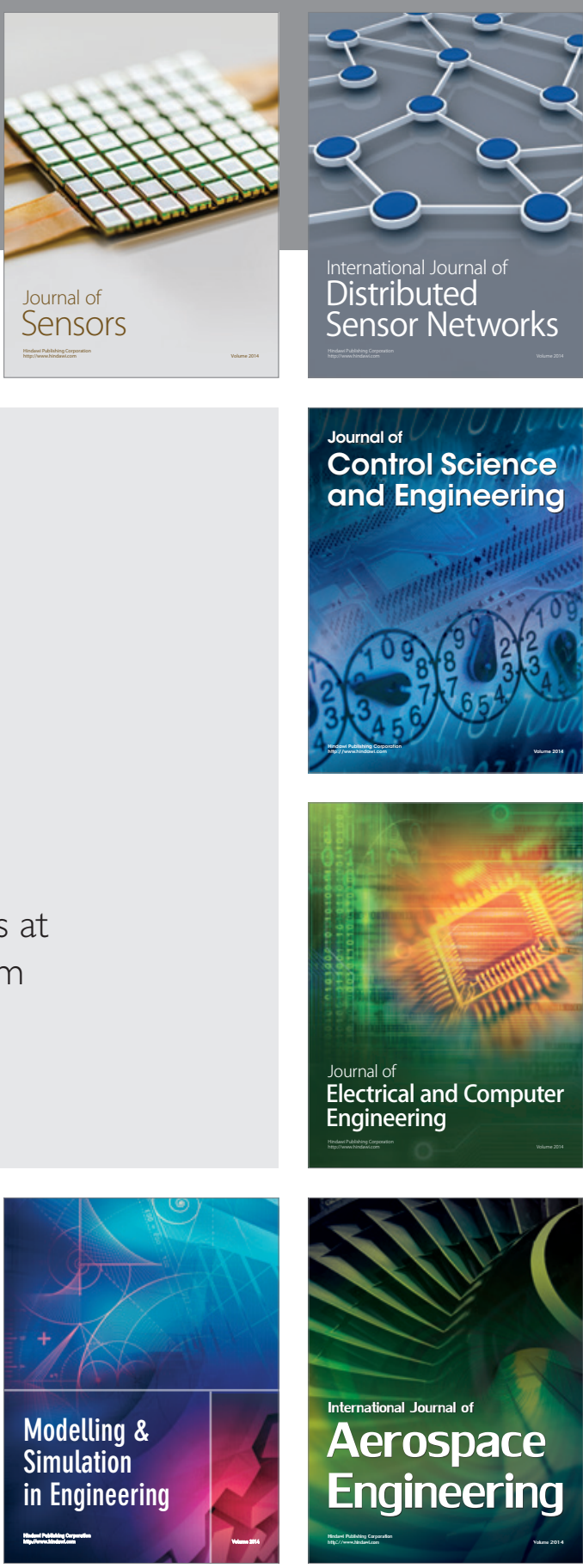

International Journal of

Distributed

Sensor Networks

Journal of

Control Science

and Engineering
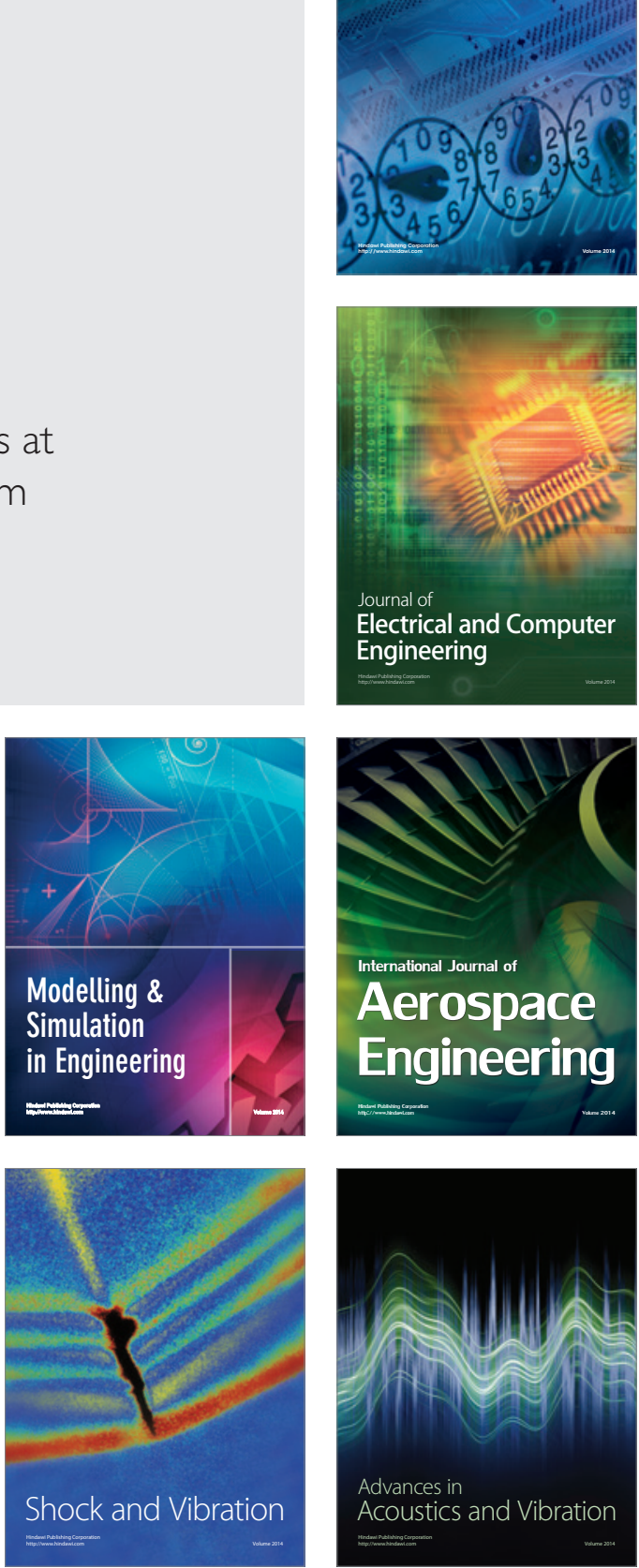